\title{
Cognitive-behavioural intervention for depression after stroke: Five single case studies on effects and feasibility
}

\author{
Citation for published version (APA):
}

Rasquin, S. M. C., van der Sande, P. A. H. F., Praamstra, A. J., \& van Heugten, C. M. (2009). Cognitivebehavioural intervention for depression after stroke: Five single case studies on effects and feasibility. Neuropsychological Rehabilitation, 19(2), 208-222. https://doi.org/10.1080/09602010802091159

Document status and date:

Published: 01/01/2009

DOI:

10.1080/09602010802091159

\section{Document Version:}

Publisher's PDF, also known as Version of record

\section{Document license:}

Taverne

Please check the document version of this publication:

- A submitted manuscript is the version of the article upon submission and before peer-review. There can be important differences between the submitted version and the official published version of record.

People interested in the research are advised to contact the author for the final version of the publication, or visit the DOI to the publisher's website.

- The final author version and the galley proof are versions of the publication after peer review.

- The final published version features the final layout of the paper including the volume, issue and page numbers.

Link to publication

\footnotetext{
General rights rights.

- You may freely distribute the URL identifying the publication in the public portal. please follow below link for the End User Agreement:

www.umlib.nl/taverne-license

Take down policy

If you believe that this document breaches copyright please contact us at:

repository@maastrichtuniversity.nl

providing details and we will investigate your claim.
}

Copyright and moral rights for the publications made accessible in the public portal are retained by the authors and/or other copyright owners and it is a condition of accessing publications that users recognise and abide by the legal requirements associated with these

- Users may download and print one copy of any publication from the public portal for the purpose of private study or research.

- You may not further distribute the material or use it for any profit-making activity or commercial gain

If the publication is distributed under the terms of Article $25 \mathrm{fa}$ of the Dutch Copyright Act, indicated by the "Taverne" license above, 


\section{Neuropsychological Rehabilitation}

\section{Cognitive-behavioural intervention for depression after stroke: Five single case studies on effects and feasibility}

\section{S. M. C. Rasquin , P. Van De Sande , A. J. Praamstra \& C. M. Van Heugten}

To cite this article: S. M. C. Rasquin , P. Van De Sande , A. J. Praamstra \& C. M. Van Heugten (2009) Cognitive-behavioural intervention for depression after stroke: Five single case studies on effects and feasibility, Neuropsychological Rehabilitation, 19:2, 208-222, DOI: $10.1080 / 09602010802091159$

To link to this article: https://doi.org/10.1080/09602010802091159

曲 Published online: 25 Feb 2009.

Submit your article to this journal $\sqsubset$

Џll Article views: 1033

Q View related articles ¿

4 Citing articles: 5 View citing articles 단 


\title{
Cognitive-behavioural intervention for depression after stroke: Five single case studies on effects and feasibility
}

\author{
S. M. C. Rasquin ${ }^{1,2,3}$, P. van de $\operatorname{Sande}^{3}$, A. J. Praamstra ${ }^{1,3,4}$, \\ and C. M. van Heugten ${ }^{1,2,3,4}$ \\ ${ }^{1}$ Vilans, Utrecht, The Netherlands; ${ }^{2}$ Department of Psychiatry and \\ Neuropsychology, Maastricht University, European Graduate School of \\ Neuroscience (EURON), Maastricht, The Netherlands; ${ }^{3}$ Rehabilitation \\ Foundation Limburg, The Netherlands; ${ }^{4}$ Rehabilitation Centre De Hoogstraat \\ Utrecht, The Netherlands
}

The purpose of the study was to investigate whether or not a cognitivebehavioural intervention for depression after stroke has an effect and is feasible. A single-subject quasi experimental design (SSED) was used with an $A B$ design and follow-up. The participants were five first episode stroke patients attending outpatient rehabilitation in a rehabilitation centre in The Netherlands. Mood and quality of life were measured on four occasions over four weeks (baseline phase A). During the eight week intervention phase (B) a visual analogue measure of mood was administered three times a week. Immediately after the intervention, and one and three months later, the baseline measures were repeated. The intervention (phase B) was based on cognitive-behavioural principles: recognising negative thoughts and challenging them, learning principles of relaxation, and planning of pleasurable activities. Following intervention three patients reported they had improved, three patients reported a minor improvement in quality of life, and four patients reported a more positive mood. Three months later three patients reported fewer depressive symptoms. Both patients and therapist were positive about the intervention and three months later, in daily life, all patients still applied the strategies. It was

Correspondence should be sent to Sascha Rasquin, SRL, PO Box 88, 6430 AB Hoensbroek, The Netherlands. E-mail: s.rasquin@srl.nl

We would like to thank the psychological assistants, M. Ploum, M. Eussen, and A. Boschloo for their participation in the data collection. The authors would like to thank Professor Wade, for his thoughtful review of this manuscript.

(C) 2008 Psychology Press, an imprint of the Taylor \& Francis Group, an Informa business http://www.psypress.com/neurorehab

DOI:10.1080/09602010802091159 
concluded that despite some ambiguous results, it seems that the cognitivebehavioural intervention has an effect on patients' mood. The intervention was rated as feasible by both patients and therapists.

Keywords: Cognitive-behavioural therapy; Stroke; Depressive symptoms; Intervention; Single case.

\section{INTRODUCTION}

Depression is one of the most troublesome consequences of stroke. Although the prevalence of depression after stroke varies between studies due to methodological differences, at least a quarter of patients develop depression after stroke (Aben, 2004; Khan, 2004; Whyte, Mulsant, Vanderbilt, Dodge, \& Ganguli, 2004). Post-stroke depression is associated with a less good rehabilitation outcome, more severe cognitive deficits and an increased risk of death (Rasquin, Lodder, \& Verhey, 2005; Robinson, Bolla-Wilson, Kaplan, Lipsey, \& Price, 1986). This makes post-stroke depression a clinically relevant problem.

Studies on treatment of post-stroke depression have mostly focused on medication interventions (Hackett, Anderson, \& House, 2004, 2005). Psychological interventions have been less frequently studied. Treatment of depressive symptoms based on cognitive-behavioural psychological principles has been proven to be effective in other chronic illnesses such as cancer or diabetes (Cuijpers, 1998). In one psychological intervention for post-stroke depression aspects of identification and challenging negative thoughts, planning of joyful activities, and behavioural activities were discussed with the patients (Lincoln, Flanaghan, Sutcliffe, \& Rother, 1997). The research group who investigated the effectiveness of this psychological intervention found conflicting results with respect to the outcome of the treatment. Four patients consistently showed beneficial treatment effects, six patients showed some benefit and nine no benefit. For the group as a whole, there was a significant decrease in depression during the treatment period on a depression scale, but no significant change in functional abilities (Lincoln et al., 1997).

This positive finding was not replicated in a larger randomised controlled trial (Lincoln \& Flannaghan, 2003). In this larger study, 39 patients received cognitive-behavioural intervention; 43 were in the attention placebo condition and 41 received standard care. There were no significant differences between the groups in terms of patients' mood, independence in instrumental activities of daily life, handicap, or satisfaction with care. In a randomised waiting-listed control group study with patients with mild and moderate traumatic brain damage living in the community (11 in the treatment group and 9 controls), individual cognitive-behavioural psychotherapy combined with individual cognitive remediation seemed to diminish psychological distress and improve 
cognitive functioning (Tiersky et al., 2005). However, it was not possible to determine which of the two interventions produced the beneficial outcome.

Several studies have investigated the possibility of preventing depression after stroke (Bédard et al., 2003; Forster \& Young, 1996; Goldberg \& Berger, 1988; Hackett et al., 2004, 2005; Watkins et al., 2007). In these studies, a small but significant reduction in psychological distress was seen using a variety of health questionnaires, but not according to standard depression diagnoses. The interventions in these studies were not based on psychological models, but were mostly based on a form of contact by other disciplines (for instance interviews by telephone) and providing information and support.

The presence of depressive symptoms increases the risk of developing a depressive disorder (Cuijpers \& Smit, 2004). Treating these depressive symptoms in an early stage after stroke might reduce the development of more severe symptoms but there is little information available on the feasibility of cognitive-behavioural intervention for stroke patients.

The purpose of this study was to investigate whether a cognitive-behavioural intervention, especially adapted for depression after stroke, has an effect and whether it is feasible. The goal of the intervention "Coping with depressive complaints after stroke" is to teach patients how to recognise negative thoughts and to challenge them, learning principles of relaxation and performing pleasant activities. This cognitive-behavioural intervention is based on the assumption that depression is either caused by or maintained by depressive thought patterns and a lack of active, positively reinforcing behavioural patterns. The intervention includes four components: self-registering of mood, changing negative thoughts (Anson \& Ponsford, 2006a), undertaking pleasurable activities, and relaxation (Anson \& Ponsford, 2006b; Bédard et al., 2003; Lai et al., 2006).

\section{METHODS}

\section{Subjects}

Stroke patients with depressive complaints were recruited in March and April 2005 from the Hoensbroeck Rehabilitation Centre in The Netherlands. If, during the rehabilitation period, the stroke patients complained about depressed feelings, they were eligible for the study. At this point inclusion and exclusion criteria were checked in more detail. The inclusion criteria were: (1) Admitted to the rehabilitation centre for stroke between January and April 2005; (2) sufficient cognitive abilities to follow the intervention, based on the clinical judgement of a psychologist, with a Mini-Mental State Examination score of at least 15 (to make sure that the patient could understand the content of the intervention); (3) report of at least one 
emotional complaint on the CheckList for Cognitive and Emotional consequences of stroke CLCE-24; and (4) age at least 18 years old.

The exclusion criteria were: (1) Insufficient understanding of the Dutch language; (2) severe aphasia (the patient had to be able to understand instructions); (3) insufficient physical endurance to participate in the sessions; (4) serious vision or hearing problems (the patient had to be able to read the information in the intervention book); (5) little or no insight into psychological functioning, based on clinical judgement; (6) co-morbid neurological disorders other than stroke; and (7) suffering or have suffered from a serious psychiatric disorder in the last five years for which patients were treated for more than half a year by a psychiatrist.

A total of 19 patients were eligible for participation in the study. Of these, seven complained about depressed feelings in their contact with the rehabilitation team. One patient refused participation and one patient had insufficient insight into his psychological functioning.

The study was approved by the medical ethics committee. All patients gave written informed consent.

\section{Measurements}

The CheckList for Cognitive and Emotional consequences following stroke (CLCE-24) was administered to assess the degree of depressive complaints. A high score represents a high number of cognitive and emotional problems which may hinder daily life (van Heugten, Rasquin, Winkens, Beusmans, \& Verhey, 2007).

Sex, age, education, current therapies and changes during the study, type and side of stroke (haemorrhage vs. infarct, right vs. left), Barthel Index (Collin, Wade, Davies, \& Horne, 1988) at admission, neuropsychological screening results (van Heugten, Huygelen, \& van de Sande, 2004), and changes in medication during the study were recorded. Neuropsychological screening consists of tests for memory (Rivermead Behavioural Memory Test), neglect (letter cancellation task), Speed of Information Processing (Trail Making Test), divided attention (Trail Making Test) and planning activities (Tower of London) (Lezak, 2004).

The Beck Depression Inventory - Second edition (BDI-II Dutch edition; van der Does, 2002) was administered to assess the degree of depression. A high score represents a high degree of complaints. Cut-off scores are: $0-13$ (minimal depression), 14-19 (mild depression), 20-28 (moderate depression), and 29-63 (severe depression).

The Symptom Checklist Depression Scale (SCL-90-D; Arrindell \& Ettema, 2003) was administered to assess the degree of depressive complaints. A high score represents more depressive complaints. The cut-off score for depression is $24 / 25$. 
The Stroke-Adapted Sickness Impact Profile-30 (SA-SIP30; van Straten et al., 1997) was administered to assess quality of life (QOL). A high score represents low QOL.

The degree of positive mood was assessed with a vertical $10 \mathrm{~cm}$ Visual Analogue Scale (VAS; Arruda, Stern, \& Legendre, 1996). A high score represents a positive mood. The upper anchor was defined as "I feel very happy", the lower anchor was defined as "I feel very unhappy". There were no middle anchors. Change of mood was measured by assessing the VAS repeatedly (see design).

A feasibility questionnaire was used to assess the experiences of the patient and the psychologist. Questions about the content, intensity, duration, and the timing of the intervention were asked. The therapist filled in the questionnaire for each patient separately and rated whether the intervention was feasible for that individual. Also the patient filled in whether he used the strategies learned in everyday life.

\section{Intervention}

The intervention was based on general cognitive-behavioural principles. We modified an existing cognitive-behavioural intervention for patients with chronic disease (Cuijpers, 1995). Most stroke patients have cognitive deficits and the modifications took this into account; the intervention book was well structured, did not contain too much information, and the information presented was easy to understand. During the first session, the intervention was introduced to the patient, the programme explained and patients were taught to record their mood. During the following two treatment sessions, the results of mood recordings were evaluated with the patients and their implications considered. Moreover, in sessions 2, 3 and 4, attention was mostly focused on relaxation by means of active relaxation exercises and visualising images of relaxation. Cognitive restructuring was started in session 4 and was elaborated in sessions 5 and 6 using situations which the patient brought to the session. The last two sessions were spent on planning useful and enjoyable activities. The last session was also used to evaluate the intervention and to find out which strategies had been most useful to the patient.

The intervention lasted eight weeks with a one hour therapeutic session with a psychologist each week. Patients also performed homework, and twice a week a psychological assistant contacted the patient to offer help performing homework. The homework consisted of recognising negative thoughts, and doing relaxation exercises and performing pleasant activities. At the start of the intervention patients received an intervention book containing theoretical background and a diary where they could record their mood and antecedent events. One therapist gave the 
intervention, and for each patient the same procedure and protocol was offered. The psychologist who offered the intervention followed the protocol strictly and each patient received the same amount of information, within the same time span.

\section{Design}

A single subject quasi experimental design was chosen. Phase A represented the baseline (four weeks) and was the test period preceding the intervention, with weekly measurements. Weekly assessment consisted of depressive complaints (BDI), mood (VAS), and quality of life (SA-SIP30). In week 1 and 4, the SCL-90 was also administered. During phase B (intervention) patients filled in the VAS three times a week. Half way through the intervention (week 5) and directly after the B phase the same tests were administered. This was repeated one month after the intervention. BDI was also repeated at three months after the intervention.

A psychologist who was unaware of the results on the questionnaires offered the intervention. The questionnaires were administered by a psychological assistant (for procedure of questionnaires, see Table 1). This psychological assistant had no information about the intervention, but simply helped the patient to fill in the questionnaires, and was told not to talk about the intervention. The time she spent with each patient was comparable between patients. A psychology student calculated the mood scores on the questionnaire and did not discuss these scores with the psychological assistant.

TABLE 1

Procedure assessment of questionnaires

\begin{tabular}{lll}
\hline Week & Assessment & \multicolumn{1}{c}{ Questionnaire } \\
\hline 1 & Baseline & BDI, Sa-SIP30, VAS, SCL-90 \\
2 & Baseline & BDI, Sa-SIP30, VAS \\
3 & Baseline & BDI, Sa-SIP30, VAS \\
4 & Baseline & BDI, Sa-SIP30, VAS, SCL-90 \\
$5-7^{*}$ & Intervention & VAS \\
$8^{*}$ & Intervention & BDI, Sa-SIP30, VAS, SCL-90 \\
$9-11^{*}$ & Intervention & VAS \\
$12^{*}$ & Intervention & BDI, Sa-SIP30, VAS, SCL-90 \\
16 & Follow-up & BDI, Sa-SIP30, VAS, SCL-90
\end{tabular}

BDI: Beck Depression Inventory; Sa-SIP30: Stroke Adapted Sickness Profile 30 items; VAS: Visual Analogue Scale; SCL-90: Symptom Checklist 90 items.

*Besides the above assessments, the VAS was completed three times a week. 
Directly after the intervention both the patient and the therapist filled in the feasibility questionnaire. Three months after the intervention the patients again filled in a questionnaire about whether or not they felt that the intervention was of help for them and whether they still used some of the principles of the intervention.

\section{Statistical analysis}

Characteristics of the patients and scores on the outcome measures were analysed using descriptive statistics. Data were plotted graphically for visual assessment of change. The Reliable Change Index (RCI) was used to define clinically significant change (Jacobson \& Truax, 1991). The RCI determines if a change observed in an individual test score is clinically significant rather than just a fluctuation caused by an imprecise measuring instrument or by chance error. The amount of change between two assessment times is divided by the standard measurement error for the scores of difference (this can be conceived as the random error in sample surveys). In accordance with the hypothesis that complaints decrease after the intervention, an RCI value smaller than $z=-1.65$ was used as a criterion for a clinical significant decrease in depressive complaints ( $p<.05$, one-sided test). The RCI could only be assessed for those questionnaires for which normative data were available (BDI and SCL-90-D). The normscores for the Dutch population of the SA-SIP30 and the VAS were not available and so an RCI could not be calculated. For the calculation of the RCI, the scores at 4 and 8 weeks and 3 months after the start of the intervention were compared with the mean baseline scores before the start of the intervention. VAS scales were measured with a ruler, and scores were plotted on a graph. Comments about feasibility were examined qualitatively.

Our single case study required a baseline in which there is no change in the outcome measure (Goodwin, 1998). The four baseline scores were considered "no change" when scores on the BDI and SCL-90-D did not change according to the RCI during the baseline period. Also after the baseline period, clinically significant changes in depressive complaints were calculated using the RCI, based on the BDI and SCL-90-D scores during and after the intervention, compared to the baseline scores. An increase of quality of life was defined as a decrease of the SA-SIP30 score in week 12 or 16 compared to the average SA-SIP30 baseline score. Mood outcomes were twofold. Firstly, progress in mood was assumed if the majority of the 12 weekly average VAS scores were higher than the average VAS baseline score. Secondly, progress in mood was defined as an increase in VAS score in week 12 or 16 compared to average VAS baseline score. 


\section{RESULTS}

\section{Subjects}

Seven patients were included, and five of them were followed during the entire research period. One patient dropped out during baseline because he had cardiac surgery. One other patient dropped out in the second week of the intervention after the psychologist decided that continuation would not be useful due to a severe lack of insight into the psychological problems which interfered with the intervention. The characteristics of patients who completed the study are presented in Table 2. All (except one) patients lived at home during the intervention and received day care at the outpatient rehabilitation clinic. Although some patients had cognitive problems, cognitive functioning was sufficient to participate. The intervention started at a mean of 7.4 months after stroke ( $S D=1.9$, range 6-10 months).

\section{Mood changes}

During the intervention patients received other therapies, such as physical therapy and occupational therapy. There was no change in the frequency of these other therapies. Patients did not have other contacts with the psychologist. However, patient three started using antidepressant medication in week 6 . As the effect of antidepressiant medication starts after 2 weeks, this probably did not interfere with the results. If there were major life events (such as a wedding or funeral), they were recorded and if necessary incorporated into the therapeutic discussion. The results of the scores on the mood outcome measures during baseline, at week 4 of the intervention, at week 8 of the intervention and 4 weeks after the end of the intervention, are presented in Figure 1.

TABLE 2

Patient characteristics

\begin{tabular}{|c|c|c|c|c|c|c|c|c|}
\hline & Sex & Age & Education & Stroke type/hemisphere & $B I(0-20)$ & Med & CLCE-24* & $\begin{array}{l}\text { Cognitive } \\
\text { problems }\end{array}$ \\
\hline 1 & $\mathrm{~F}$ & 44 & Medium & Infarct/right & 10 & $\mathrm{Y}$ & 5 & $1,2,3$ \\
\hline 2 & $\mathrm{~F}$ & 48 & Medium & Infarct/right & 20 & $\mathrm{~N}$ & 6 & 1,3 \\
\hline 3 & $\mathrm{~F}$ & 46 & Low & Infarct/right & 13 & $\mathrm{Y}$ & n.a. & 3,4 \\
\hline 4 & $\mathrm{~F}$ & 39 & Medium & Haemorrhage/cerebellum & 18 & $\mathrm{~N}$ & 5 & 3,4 \\
\hline 5 & M & 54 & Low & Infarct/right & 14 & $\mathrm{Y}$ & 5 & 3 \\
\hline
\end{tabular}

n.a.: not available; $1=$ neglect, $2=$ impulsivity, $3=$ low speed of information processing, $4=$ problems with divided attention; $\mathrm{BI}=$ Barthel index at admission to rehabilitation centre; Med $=$ antidepressant medication; CLCE: CheckList for Cognitive and Emotional consequences of stroke.

${ }^{*}$ Number of problems. 
(a)

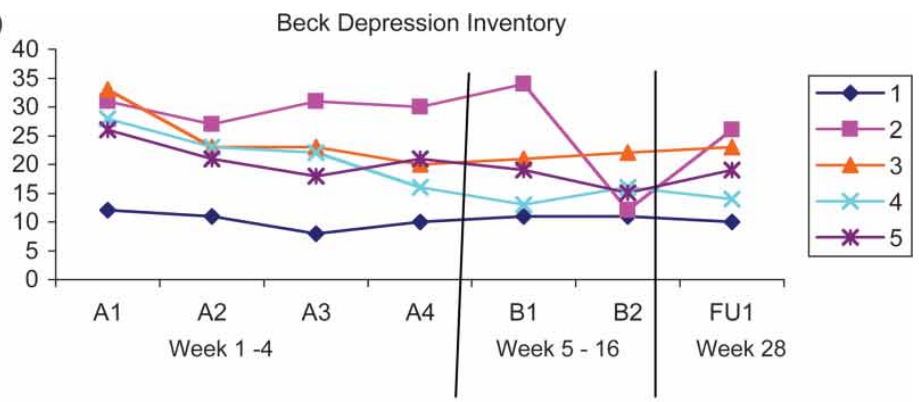

(b)

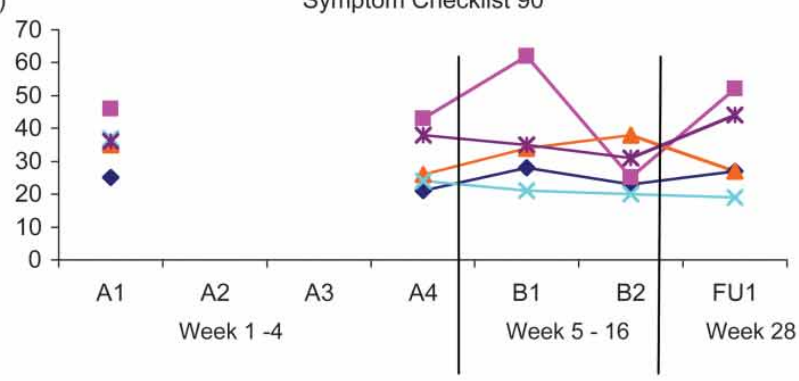

(c) Stroke Adapted Sickness Impact Profile

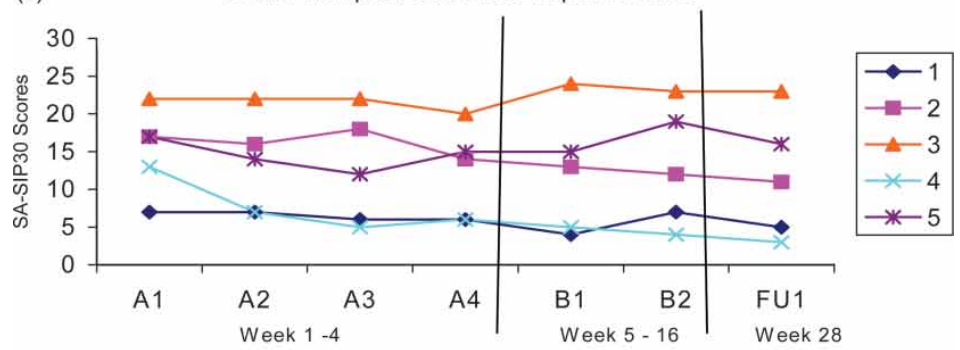

(d) Visual Analogue Scale

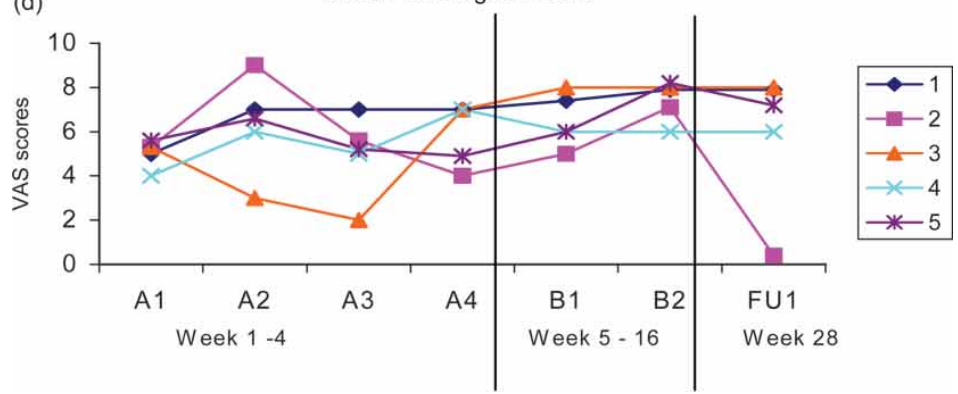

Figure 1. (a-d) Scores on the mood scales per individual, over time. A1, A2, A3, A4: assessment during 4 week baseline (once a week), B1, B2: assessment during 8 week intervention (once a month), FU1: assessment 3 months after end of the intervention, numbers 1 to 5: patient numbers 
Overall, the baseline measures were stable for all patients. For patients three, four, and five there were some fluctuations in the BDI score during baseline. However, these fluctuations were only between two assessments, and overall there was no change.

Four and eight weeks after the start of the intervention, patients two, four and five showed clinically significant improvement on the BDI. Patient two improved from a mean BDI baseline score of $29.8(S D=1.9)$ to a score of 12 after the end of the intervention, patient four from $22.3(S D=4.9)$ to 16 , and patient five from $21.5(S D=3.3)$ to 15 . Patients one and three did not change.

On the SCL-90 all patients except patient two (who improved) did not change. Patient two improved from a mean SCL-90 baseline score of 44.5 to 25 at the end of the intervention. On the SA-SIP30 two patients (two and four) improved, patients one, three and five did not change. Patient two improved from a mean SA-SIP baseline score of 16.3 to a score of 12 at the end of the intervention, patient four improved from 7.8 to a score of 4 after the intervention. All patients improved on the VAS.

One month after the intervention none of the patients changed compared to the end of the intervention, except for patient two who had a decline in VAS score. Three months after the intervention, only the BDI was assessed; only patient two improved, and the others remained stable.

\section{Visual Analogue Scale}

In addition to the VAS scores at 4, 8 and 12 weeks after start of the intervention, during the intervention patients also filled in a VAS three times a week. Weekly mean (over three days) scores are presented in Figure 2.

Weekly mean VAS scores of patient one improved at week 3 ( 0.5 points), week 4 (0.9 points), week 7 (0.3 points), and week 8 (1.5 points) of the

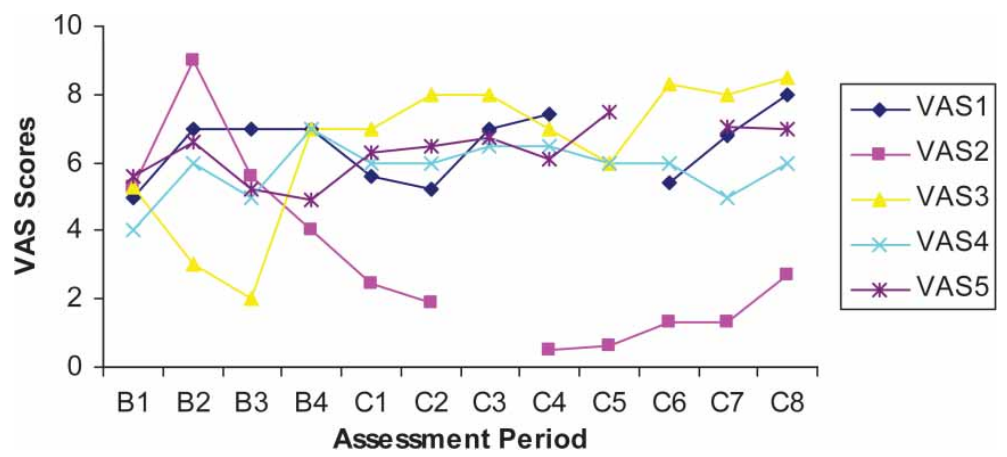

Figure 2. Mean weekly VAS scores 
intervention, compared to mean baseline score (mean $=6.5$ ). In weeks 1,2 , and 3 of the intervention the weekly mean VAS score was less than the mean baseline score (respectively 1 point, 1.3 points, and 1 point). In week 5 of the intervention no VAS was filled in.

Weekly mean VAS scores of patient two in weeks 1-8 were less (all were more or less 1 point less) than the mean baseline score (mean $=5.9$ ), although there was an increasing trend in VAS scores after week 4 of the intervention. In week 3 of the intervention no VAS was filled in.

The weekly mean VAS score of patient three improved from week 1 to week 8 (a mean improvement of 3.3 points) of the intervention, compared to the mean baseline score (mean $=4.3$ ).

The weekly mean VAS score of patient four improved (an improvement ranging from $0.5-1$ point) in all weeks of the intervention, compared to the mean baseline score (mean $=5.5$ ). Except in week 7, the weekly mean VAS score was less than the mean baseline score.

The weekly mean VAS score of patient five improved by one point from week 1 to week 8 of the intervention compared to the mean baseline score (mean $=5.6)$. In week 6 of the intervention the VAS was not filled in.

\section{Feasibility questionnaire}

Directly after the intervention, patients filled in the feasibility questionnaire. All patients were positive about the intervention (mean score was $7.4 ; S D 0.5$; range 7.0-8.0). One patient would have preferred to receive the intervention earlier after stroke and one would have preferred the informal caregiver to be more involved. For one patient the number of meetings was too high, especially the scientific assessments (which followed directly after the intervention). The psychologist who gave the intervention was also positive (mean 7.0; $S D$ 0.5; range 6.0-8.0). However, he was more critical than the patients. He pointed out that for some $(n=2)$ patients the intervention was too early, and that for two patients the relaxation principles were not applicable as they used other forms of relaxation.

All patients said that they used the strategy in everyday life. Both the patients and the therapist expected that all the patients would apply the methods learned in the intervention in future. Three months after the end of the intervention, this was indeed the case. Moreover, all patients thought that the intervention had helped them to handle mood problems.

\section{DISCUSSION}

The main purpose of this study was to investigate whether a cognitive behavioural intervention for depressive complaints had an effect on mood and was feasible for stroke patients. Based on the results we can conclude that a 
cognitive-behavioural intervention is feasible for a subgroup of patients who receive rehabilitation care due to stroke and who complain about depressive mood. Two patients could not complete the intervention; one because of cardiac surgery and one had a too severe lack of insight into his psychological problems. Whereas this last patient should not have been included, we thought it was necessary to report this patient as it stresses the importance of good insight into emotional functioning. Although the intervention seems feasible, we cannot firmly conclude that this intervention reduces mood complaints. Ambiguous results were found in relation to mood changes. Not all patients improved and substantial emotional complaints remained (although the number of symptoms was lower). Four weeks after the end of the intervention, four patients had the same scores compared to the assessment at the end of the intervention which was overall better compared to the baseline scores. One patient had an increase in emotional complaints which could be attributed to medical problems (migraine and bursitis) during the follow-up period. After finishing the intervention, all patients still practised what they had learned. All patients and the psychologist were positive about the intervention.

Based on the results and clinical experience, we evaluated that the intervention may not have been adequate in quantity. The therapist felt that the intervention should contain more sessions and should focus more on performing pleasurable activities. Moreover, as patients with stroke often have a paralysis, relaxation of the body should be an essential component of the intervention. These changes will be investigated in a future study.

None of the patients had an increase of emotional complaints, even when they experienced serious life events. For instance, patient two decreased substantially on the VAS during the baseline, but showed a trend towards an increase of positive mood during the intervention. This decrease could be attributed to the discharge home. This patient had to encounter many difficulties due to the independent life she had to live. In spite of these problems her mood improved during the intervention. Based on these results we could cautiously conclude that the psychological intervention prevents increase of depressive symptoms. Other studies have also found improved mood after being treated with cognitive-behavioural therapy, although these were in patients seen much later after stroke.

The results of this study are comparable to the results of the pilot study of Lincoln et al. (1997), which were not replicated in a randomised controlled trial in a larger group of patients possibly because all depressive stroke patients were included, even the patients with too little insight or with complex cognitive deficits who might have less potential to benefit from the intervention.

Other studies investigating comparable interventions have found improvement in mood or engagement in social activities (Forster \& Young, 1996; 
House, Dennis, Warlow, Hawon, \& Molyneux, 1990). It seems that therapies based on cognitive-behavioural principles are feasible and have a positive effect on the mood of stroke patients. Nevertheless further studies are needed before firm conclusions can be stated, especially in the light of the only large randomised controlled trial.

This study has its shortcomings. First, the design is a single case design and generalisation to the entire stroke population with depressive symptoms is not possible. Unfortunately randomising the length of baseline, which would have increased control, was not possible for practical reasons, and so we used a baseline period of 4 weeks. Second, with this design it is more difficult to control for confounding effects such as other therapies, medication use and the impact of emotional events such as a divorce. To reduce these confounding factors to a minimum, the rehabilitation team was asked not to change the patients' other therapies.

Third, the follow-up period of this study was short. In this intervention patients learned to recognise negative thoughts and challenge them and learned the principles of relaxation and to perform pleasant activities. It is possible that the effects of the intervention only became apparent after a longer period, when the patients had had enough time to use the strategies learned.

Fourth, we had no control group or control phase. However, as the goal of this study was to get some information about the feasibility and effect of the cognitive-behavioural therapy on stroke patients, a control group or control phase would be too expensive and time-consuming in this research stage. Since this study indicates that cognitive-behavioural therapy is feasible and has an effect on mood, a next study should be conducted incorporating a control group.

Based on these results we can conclude that this intervention is feasible but inadequate in "dose", and we can cautiously conclude that the intervention might reduce depressive symptoms. The results justify future research about the effectiveness of this intervention in a larger group.

\section{REFERENCES}

Aben, I. (2004). Post-Stroke Depression. The Need for a Bio-Psycho-Social Approach. Doctoral Thesis University Maastricht.

Anson, K., \& Ponsford, J. (2006a). Coping and emotional adjustment following traumatic brain injury. Journal of Head Trauma Rehabilitation, 21(3), 248-259.

Anson, K., \& Ponsford, J. (2006b). Evaluation of a coping skills group following traumatic brain injury. Brain Injury, 20(2), 167-178.

Arrindell, W. A., \& Ettema, J. H. M. (2003). Symptom Checklist [Handleiding bij een multidimensionele psychopathologie-indicator]. Lisse: Swets \& Zeitlinger. 
Arruda, J. E., Stern, R. A., \& Legendre, S. A. (1996). Assessment of mood state in patients undergoing electroconvulsive therapy: The utility of Visual Analog Mood Scales developed for cognitively impaired patients. Convulsive Therapy, 12(4), 207-212.

Bédard, B. V. M., Felteau, M., Mazmamian, D., Fedyk, K., Klein, R., Richardson, J., et al. (2003). Pilot evaluation of a mindfulness-based intervention to improve quality of life among individuals who sustained traumatic brain injuries. Disability Rehabilitation, 25(13), 722-731.

Collin, C., Wade, D. T., Davies, S., \& Horne, V. (1988). The Barthel ADL Index: A reliability study. International Disability Studies, 10(2), 61-63.

Cuijpers, P. (1995). The course: Handling depression. [De curus omgaan met depressie]. Gedragstherapie, 28(3),189-208.

Cuijpers, P. (1988). Prevention of depression in chronic general medical disorders: A pilot study. Psychology Reports, 82, 735-738.

Cuijpers, P., \& Smit, F. (2004). Subthreshold depression as a risk indicator for major depressive disorder: A systematic review of prospective studies. Acta Psychiatrica Scandinavica, 109(5), 325-331.

Forster, A., \& Young, J. (1996). Specialist nurse support for patients with stroke in the community: A randomised controlled trial. British Medical Journal, 312(7047), 1642-1646.

Goldberg, G., \& Berger, G. G. (1988). Secondary prevention in stroke: A primary rehabilitation concern. Archives of Physical Medical Rehabilitation, 69(1), 32-40.

Goodwin, C. J. (1988). Research in psychology: Methods and design (2nd ed.). New York: John Wiley \& Sons.

Hackett, M. L., Anderson, C. S., \& House, A. O. (2004). Interventions for treating depression after stroke. Cochrane Database Systematic Review, 3, CD003437.

Hackett, M. L., Anderson, C. S., \& House, A. O. (2005). Management of depression after stroke: A systematic review of pharmacological therapies. Stroke, 36(5), 1098-1103.

House, A., Dennis, M., Warlow, C., Hawon, K., \& Molyneux, A. (1990). The relationship between intellectual impairment and mood disorder in the first year after stroke. Psychological Medicine, 20(4), 805-814.

Jacobson, N. S., \& Truax, P. (1991). Clinical significance: A statistical approach to defining meaningful change in psychotherapy research. Journal of Consulting and Clinical Psychology, 59(1), 12-19.

Khan, F. (2004). Post-stroke depression. Australian Family Physician, 33(10), 831-834.

Lai, S. M., Studenski, S., Richards, L., Perera, S., Reker, D., Rigler, S., \& Duncan, P. W. (2006). Therapeutic exercise and depressive symptoms after stroke. Journal of the American Geriatric Society, 54(2), 240-247.

Lezak, M. D. (2004). Neuropsychological Assessment (3rd ed.). New York: Oxford University Press.

Lincoln, N. B., Flanaghan, T., Sutcliffe, L., \& Rother, L. (1997). Evaluation of cognitive behavioural treatment for depression after stroke: A pilot study. Clinical Rehabilitation, 11(2), $114-122$.

Lincoln, N. B., \& Flannaghan, T. (2003). Cognitive behavioral psychotherapy for depression following stroke: A randomized controlled trial. Stroke, 34(1), 111-115.

Rasquin, S. M. C., Lodder, J., \& Verhey, F. R. J. (2005). The influence of psychiatric symptoms on cognitive performance after stroke. Cerebrovascular Disease, 19(5), 309-316.

Robinson, R. G., Bolla-Wilson, K., Kaplan, E., Lipsey, J. R., \& Price, T. R. (1986). Depression influences intellectual impairment in stroke patients. British Journal of Psychiatry, 148, $541-547$.

Tiersky, L. A., Amselmi, V., Johnston, M. V., Kurkyka, J., Roosen, E., Schwartz, T., \& Deluca, J. (2005). A trial of neuropsychologic rehabilitation in mild-spectrum traumatic brain injury. Archives of Physical Medical Rehabilitation, 86(8), 1565-1574. 
van der Does, A. J. W. (2002). BDI-II-NL Manual. The Dutch version of the Beck Depression Inventory (2nd ed.). Lisse: Swets \& Zeitlinger.

van Heugten, C. M., Huygelen, K., \& van de Sande, P. (2004) [Cognitive screening in stroke patients in rehabilitation: Standards for clinical practice]. Tijdschrift Gerontologie Geriatrica, 35(5), 196-202.

van Heugten, C. M., Rasquin, S. M. C., Winkens, I., Beusmans, G., \& Verhey, F. R. J. (2007). Checklist for cognitive and emotional consequences following stroke (CLCE-24): Development and validation. Clinical Neurology and Neurosurgery, 109, 257-262.

van Straten, A., de Haan, R. J., Limburg, M., Schuling, J., Bossuyt, P. M., \& van den Bos, G. A. (1997). A stroke-adapted 30-item version of the Sickness Impact Profile to assess quality of life (SA-SIP30). Stroke, 28(11), 2155-2161.

Watkins, C. L., Auton, M. F., Deans, C. F., Dickinson, H. A., Jack, C. I., Lightbody, C. E., et al. (2007). Motivational interviewing early after acute stroke: A randomized, controlled trial. Stroke, 38(3), 1004-1009.

Whyte, E. M., Mulsant, B. H., Vanderbilt, J., Dodge, H. H., \& Ganguli, H. (2004). Depression after stroke: A prospective epidemiological study. Journal of the American Geriatric Society, 52(5), 774-778.

Manuscript received October 2007 Revised manuscript received March 2008

First published online June 2008 